\title{
Acute Hepatitis A Infection and Vaccination in the Veterans Health Administration
}

\author{
Gina Oda ${ }^{1}$, Cynthia A. Lucero-Obusan ${ }^{1}$, Patricia Schirmer ${ }^{1}$, Mark Holodniy ${ }^{1,} 2$ \\ ${ }^{1}$ Public Health Surveillance and Research, Department of Veteran Affairs, Palo Alto, California, United States, ${ }^{2}$ Stanford University Division of \\ Infectious Diseases and Geographic Medicine, Stanford, California, United States
}

\section{Objective}

To describe the epidemiology of hepatitis A virus (HAV) within the Veterans Health Administration (VHA).

Introduction

Since hepatitis A vaccination became widely recommended in the US in the mid-1990's, rates of acute hepatitis A virus (HAV) infection have steadily declined, however, since 2011, incidence of new cases of HAV appears to be increasing [1], often linked with foodborne outbreaks and socio-economic trends such as homelessness and substance abuse [2]. In 2016, the CDC reported vaccination coverage among adults aged $\geq 19$ was $9.5 \%, 19-49$ was $13.4 \%$, and $\geq 50$ was 5.4\% [3]. CDC issued a Health Alert Network Advisory in June 2018 with additional guidance on identification and prevention of HAV and updates on outbreaks in multiple states [4] which prompted our program to conduct a more formal review of HAV infections in VHA. Herein we describe recent trends in HAV infection, vaccination and associated risk factors among Veterans.

\section{Methods}

We queried VA data sources from October 1, 2016 - June 30, 2018 for HAV IgM laboratory tests, HAV-coded outpatient encounters and hospitalizations (ICD-10-CM: B15), and pharmacy data for hepatitis A vaccinations administered in VHA outpatient and inpatient settings. Patients with coded HAV encounter or hospitalization were compared to individuals with HAV IgM positive results to determine Positive Predictive Value (PPV) of HAV outpatient and inpatient diagnostic codes. A total of 30 (20 outpatient and 10 inpatient HAV encounters across both fiscal years) were randomly selected for detailed chart review to determine if patients were properly coded. Additionally, patients with positive HAV IgM results were analyzed for ICD-10-CM coded outpatient and inpatient encounters indicative of homelessness (ICD-10-CM: Z59.0) and/or substance abuse (ICD-10-CM: F1x, excluding nicotine and cannabis). Rates were calculated using total unique users of VHA care for matching fiscal year time frames and geographic area as denominators. We reviewed a sample of 10 electronic medical records (EMR) of patients from Hawaii to determine vaccine indications in the setting of a state-wide outbreak.

\section{Results}

A total of 136,970 HAV IgM tests were performed between October 1, 2016 - June 30, 2018. We identified 247 unique patients with positive HAV IgM. The overall incidence during the study time period was 2.05 per 100,000 population of unique users of VHA care. The state with the highest incidence was West Virginia (9.49 per 100,000) (Figure 1). The overall percent positivity of patients tested for HAV IgM was $0.18 \%$ (highest of $1.16 \%$ for Kentucky). There were 1,085 HAV-coded outpatient encounters (680 unique patients) but only 58 patients had a positive HAV IgM result (PPV= 8.5\%). There were 371 HAV-coded hospitalizations ( 335 unique patients) but only 39 patients had a positive HAV IgM result (PPV=11.6\%). Among these encounters, 270 outpatients had HAV documented as the principal diagnosis for the visit (40 of these were HAV IgM+) and 38 hospitalized patients had HAV as the principal discharge diagnosis code ( 25 of these were HAV IgM+). Therefore, the PPV when HAV was the principal diagnosis code improved to $14.8 \%$ for outpatient encounters and $65.8 \%$ for inpatients. Chart review of 30 randomly selected outpatient and inpatient HAV-coded EMR found that only 3 (10\%) were correctly coded. Of the remaining 27, 14 (47\%) had a positive HAV IgG or HAV Total test result, but negative or no HAV IgM testing, 3 (10\%) had a remote history of HAV, 3 (10\%) were rule-out HAV but testing was negative, 2 (7\%) were miscodes of hepatitis B (HBV) or hepatitis C Virus (HCV) infections, and 5 (17\%) were other miscodes. The median vaccination rate during the study time frame was $0.31 \%$ [range: $0.11 \%$ (Puerto Rico) to $3.48 \%$ (Hawaii)]. Additional states with vaccination rates above the median included Kentucky, Michigan, West Virginia, and California $(1.05 \%, 1.02 \%, 0.93 \%, 0.67 \%$, respectively). Review of 10 sample EMR of patients from Hawaii, the state with highest vaccination rate, indicated that during their vaccination peak, patients were receiving the $2^{\text {nd }}$ in their 2 -dose HAV 
series, the first having been given in August 2016 at the time of a state-wide outbreak associated with raw scallops. Of 247 patients with positive HAV IgM, 91 (37\%) had presence of ICD-10- CM encounter codes for one or more of the following risk factors associated with HAV outbreaks (in order of frequency): substance abuse (63/247; 26\%), homelessness (36/247; 15\%), HCV (30/247; 12\%), and HBV (2/247; 0.8\%). Wayne County, MI, Jefferson County, KY, and San Diego County, CA all had clustering of 4 or more cases of acute HAV with risk factors of homelessness, substance abuse, and HCV.

\section{Conclusions}

Acute HAV was identified in the VHA patient population in states associated with recognized outbreaks during the study time frame. Associated risk factors of substance abuse, homelessness, and HCV found in the Veteran population also matched national HAV outbreak data, including clustering in specific counties where outbreaks occurred. Overall, PPV for HAV-coded encounters was low for both inpatients and outpatients due to frequent miscoding. PPV was improved among inpatients with a principal discharge diagnosis of acute HAV. Vaccination rates were likely underestimated as data prior to the study time period was not evaluated and patients may have received vaccine outside of VA, however rates tended to be above the median in states with known outbreaks, possibly indicating ongoing response. In the case of Hawaii, EMR review indicated that a strong public health response demonstrated by a high post-outbreak vaccination rate with Veterans being monitored and brought back for their $2^{\text {nd }}$ of 2 vaccine series occurred following the August 2016 HAV outbreak associated with raw scallops. ${ }^{2}$ Additional education of VA providers is warranted regarding the timely recognition of, proper testing for, and coding of acute HAV infections and improving vaccination rates, particularly among individuals who are at increased risk for infection or complications from HAV.

\section{Acknowledgement}

The views expressed are those of the authors and do not necessarily reflect the position or policy of the Department of Veterans Affairs or the United States government.

\section{References}

1. CDC. 2016. Progress Toward Eliminating Hepatitis A Disease in the United States. MMWR Suppl. 65(1), 2941. PubMed https://doi.org/10.15585/mmwr.su6501a6

2. CDC. Hepatitis A Outbreaks. https://www.cdc.gov/hepatitis/outbreaks/hepatitisaoutbreaks.htm. Accessed September 6, 2018.

3. CDC. National Health Interview Survey, Atlanta, GA: US Department of Health and Human Services, CDC; 2016. https://www.cdc.gov/vaccines/imz- managers/coverage/adultvaxview/pubs-resources/NHIS2016.html\#hepA. Accessed September 12, 2018.

4. CDC. Health Alert Network Advisory: Outbreak of Hepatitis A Virus (HAV) Infections among Persons Who Use Drugs and Persons Experiencing Homelessness. June 11, 2018.

https://emergency.cdc.gov/han/han00412.asp. Accessed September 6, 2018. 


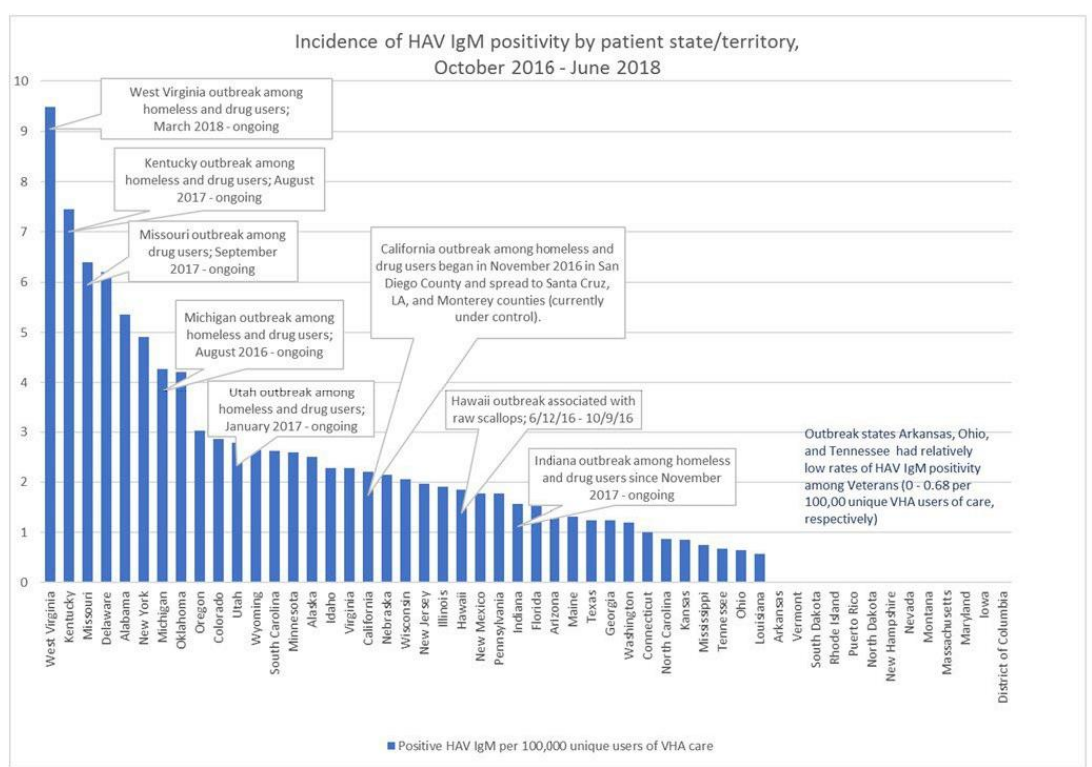

Figure 1. 